\title{
Population receptive field estimates in human visual cortex
}

\author{
Serge O. Dumoulin ${ }^{*}$ and Brian A. Wandell \\ Psychology, Stanford University, Stanford, CA 94305, USA
}

\begin{abstract}
We introduce functional MRI methods for estimating the neuronal population receptive field (pRF). These methods build on conventional visual field mapping that measures responses to ring and wedge patterns shown at a series of visual field locations and estimates the single position in the visual field that produces the largest response. The new method computes a model of the population receptive field from responses to a wide range of stimuli and estimates the visual field map as well as other neuronal population properties, such as receptive field size and laterality. The visual field maps obtained with the pRF method are more accurate than those obtained using conventional visual field mapping, and we trace with high precision the visual field maps to the center of the foveal representation. We report quantitative estimates of pRF size in medial, lateral and ventral occipital regions of human visual cortex. Also, we quantify the amount of input from ipsi- and contralateral visual fields. The human pRF size estimates in V1-V3 agree well with electrophysiological receptive field measurements at a range of eccentricities in corresponding locations within monkey and human visual field maps. The pRF method is non-invasive and can be applied to a wide range of conditions when it is useful to link fMRI signals in the visual pathways to neuronal receptive fields.
\end{abstract}

\section{Keywords}

Visual cortex; fMRI; pRF; Visual areas; V1; Neuron

\section{Introduction}

Bridging the gap between technologies that measure neural signals at different length scales is an important objective in neuroimaging research. Here, we introduce new experimental and computational methods that quantitatively couple fMRI signals, measured at the millimeter scale, with receptive field properties of visual neurons measured at the micron scale.

The new methods build on techniques that were developed for visual field mapping (DeYoe et al., 1996; Dumoulin et al., 2003; Engel et al., 1997; Engel et al., 1994; Sereno et al., 1995). In visual field mapping, the experimenter measures responses to contrast-defined rings and wedges shown at a series of visual field locations. Conventionally, the responses to these stimuli are used to estimate the visual field position that produces the largest fMRI response for each voxel.

While conventional methods estimate only the most effective visual field location, the neuronal population within a voxel in fact responds to a range of visual field locations. The 
region of visual space that stimulates the recording site is the population receptive field (pRF) (Victor et al., 1994). The pRF method estimates the visual field map, the pRF size, laterality and surround suppression using the temporal responses to multiple stimuli.

Intuitively, the pRF size can be estimated because we understand how pRF size influences the fMRI time course. This pRF influence was observed by Tootell et al. (1997), who noticed different time courses in $\mathrm{V} 1$ and $\mathrm{V} 3 \mathrm{~A}$ in response to expanding ring stimuli commonly used to map the visual field (Engel et al., 1994). They explained this time course difference by suggesting that pRF sizes in V3A exceed those of V1. Smith et al. (2001) extended this analysis by measuring the relative amount of active versus inactive epochs (duty cycle) in the fMRI response to the ring stimulus (see also Larsson and Heeger, 2006; Li et al., 2007). In addition to comparisons between areas, Smith et al. (2001) also found a systematic relationship between the duty cycle and visual eccentricity.

The advance we describe here is a quantitative framework to model pRF properties and fit these models to the fMRI time series. Specifically, we show how to derive visual field maps and $\mathrm{pRF}$ sizes by integrating data from multiple types of stimuli, including rings, wedges, and moving bars. We show that this model-based approach reconstructs the cortical visual field map more accurately than conventional mapping methods. We use the pRF method to obtain quantitative estimates of population receptive field sizes in lateral and ventral occipital regions of human visual cortex. Finally, we show that the human pRF size estimates in areas V1-V3 agree well with electrophysiological receptive field measurements in the corresponding areas in monkey and human visual cortex.

\section{Materials and methods}

\section{Subjects}

Measurements were obtained from six subjects (one female; ages 24-36 years). All subjects participated in experiments containing wedges and ring stimuli, three subjects participated in experiments containing the moving bar stimuli. All subjects had normal or corrected-tonormal visual acuity. All studies were performed with the informed written consent of the subjects and were approved by the Stanford Institutional Review Board.

\section{Stimulus presentation}

The visual stimuli were generated in the Matlab programming environment using the PsychToobox (Brainard, 1997; Pelli, 1997) on a Macintosh G4 Powerbook. Stimuli were displayed in one of two configurations. In both configurations the subjects viewed the display through an angled mirror. The first display configuration consisted of an LCD (NEC, 2080UX) housed in an electrically shielded box with conductive glass on the front side. The experiments were performed with the LCD positioned at the back of the magnet bore, behind the subject's head (distance $2.8 \mathrm{~m}$ ). The maximum stimulus radius was $3^{\circ}$ of visual angle. The second display configuration consisted of an LCD projector (NEC LT158) with optics that imaged the stimuli onto a back-projection screen in the bore of the magnet. In this configuration, the maximum stimulus radius was $14^{\circ}$ of visual angle.

\section{Stimulus description}

In one set of experiments we used conventional rotating wedge and expanding ring sections of a high-contrast, moving, dartboard pattern (DeYoe et al., 1996; Dumoulin et al., 2003; Engel et al., 1997; Engel et al., 1994; Sereno et al., 1995). The dartboard pattern is exposed by slowly moving apertures either in the shape of a rotating wedge or an expanding ring (see Figs. 1A and B). The apertures follow a periodic pattern and complete a full cycle in $24 \mathrm{~s}$ 
with a total of 6 cycles per scanning run (for a schematic diagram, see Fig. 1E). The wedge subtended $45^{\circ}$ and the rings were $1 / 4$ th of the maximum stimulus radius.

We also used a modified version of the ring and wedge stimuli that included periods in which the observer saw only a mean luminance (zero contrast) field (see Figs. 1C and F). These mean-luminance periods were inserted at a different rate $(4$ cycles/scan) than the rotating wedge or expanding ring cycles (6 cycles/scan). Using this method, each meanluminance presentation replaces a different position of the wedge or ring stimulus.

Additionally, we measured responses to drifting bar apertures at various orientations; these bar apertures exposed a checkerboard pattern (100\% contrast; Fig. 1D). The bar width subtended 1/4th of the stimulus radius. Four bar orientations and two different motion directions for each bar were used, giving a total of 8 different bar configurations within a given scan (Fig. 1D). Note that the bars are not "phase-encoded" stimuli; there is no repetition of the stimulus because the bars change orientation and motion direction within a scan.

Finally, we estimated the hemodynamic impulse response function using briefly pulsed ( $3 \mathrm{~s}$ ) full-field checkerboard patterns that were displayed six times, separated by 30 -s intervals.

\section{Magnetic resonance imaging}

Magnetic resonance images were acquired with a $3 \mathrm{~T}$ General Electric Signa scanner and a custom-designed surface coil (Nova Medical, Wilmington, MA) centered over the subject's occipital pole. Foam padding and tape minimized head motion.

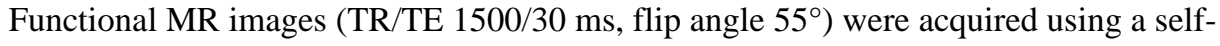
navigated spiral-trajectory pulse sequence (Glover, 1999b; Glover and Lai, 1998) with 20 slices oriented orthogonal to the Calcarine sulcus with no slice gap. The effective voxel size was $2.5 \times 2.5 \times 3 \mathrm{~mm}^{3}\left(\mathrm{FOV}=24 \times 24 \mathrm{~cm}^{2}\right)$. Functional scans using wedge or ring stimuli were acquired during 106 time frames (scan duration of $2.65 \mathrm{~min}$ ). Between 8 and 16 scans were performed in each session. Functional scans using bar stimuli measured at 138 or 202 time frames ( 3.5 or $5 \mathrm{~min}$, respectively). Functional scans to measure the HRF acquired 128 time frames (3.2 min). Between 4 and 8 functional scans were performed in each session. T1weighted anatomical MR images were acquired using a fast spoiled gradient echo (SPGR) sequence prior to the functional scans and using the same slice prescription as the functional scans.

In a separate session, high-resolution T1-weighted MRI images were acquired on a $1.5 \mathrm{~T}$ Signa LX scanner with a vendor-supplied head-coil using a 3D-SPGR pulse sequence (1 echo, minimum TE, flip angle $15^{\circ}$, effective voxel size of $0.94 \times 0.94 \times 1.2 \mathrm{~mm}^{3}$ ). We acquired at least 2 whole brain T1-weighted anatomical MRI data sets for each subject.

\section{Processing of anatomical images}

The T1-weighted anatomical MRI data sets were averaged and re-sampled to a $1 \mathrm{~mm}^{3}$ isotropic resolution. The surface-coil anatomical MRI, taken at the same time as the functional images, was aligned with the head-coil anatomical MRI using a mutual information method (Ashburner and Friston, 2003; Maes et al., 1997). The functional images and surface-coil anatomical data acquired in the same session and thus were coregistered. Using the spiral acquisition and small field of view surface-coil limits the size of the distortions between the functional and surface-coil anatomical images. Hence, we used the transformation derived from the surface-coil anatomical to align the functional data to the head-coil anatomical. 
Gray and white matter was segmented from the anatomical MRI using custom software and hand-edited to minimize segmentation errors (Teo et al., 1997). The cortical surface was reconstructed at the white/gray matter border and rendered as a smoothed 3D surface (Wandell et al., 2000).

\section{Preprocessing of functional images}

The first 8 timeframes of each functional run were discarded due to start-up magnetization transients. Head movement and motion artifacts within and between scans were measured (Nestares and Heeger, 2000). With these experienced adult subjects, the scans contained minimal head motion (less than one voxel), so no motion correction algorithm was applied.

\section{Model-based analysis}

The pRF parameters were estimated from the time series data (Fig. 2) using a linear spatiotemporal model of the fMRI response. Although this linearity fails under certain conditions, it is a reasonable approximation over a wide range of spatio-temporal conditions (Birn et al., 2001;Boynton et al., 1996; Hansen et al., 2004). Assuming a linear relationship between the blood oxygenation levels and the MR signals, $y(t)$, can be described as:

$$
y(t)=p(t) \beta+e
$$

where $p(t)$ is the predicted BOLD signal, $\beta$ is a scaling factor that accounts for the unknown units of the fMRI signal, and $e$ is measurement noise. It is common practice in neuroimaging to use a general linear model (GLM) and enter the predicted BOLD signal into a design matrix. The experimenter then estimates the response strength $(\beta)$ for each column of the design matrix (Friston et al., 1995; Worsley and Friston, 1995). In the model-based analysis developed here, the prediction $p(t)$ is calculated using a parameterized model of the underlying neuronal population and the stimulus. We estimated the neuronal model by finding the model parameters that best predict the observations. The value of this procedure is that the estimated parameters are connected meaningfully to the neuronal parameters.

We illustrate the calculation of the prediction, $p(t)$, using a Gaussian model of the neuronal population. A similar procedure can be applied using other models. A two-dimensional Gaussian pRF, $g(x, y)$, is defined by three parameters, $x_{0}, y_{0}$ and $\sigma$,

$$
g(x, y)=\exp -\left(\frac{\left(x-x_{0}\right)^{2}+\left(y-y_{0}\right)^{2}}{2 \sigma^{2}}\right)
$$

where $\left(x_{0}, y_{0}\right)$ is the center and $\sigma$ is the Gaussian spread (standard deviation). Note that these parameters are stimulus-referred; the units of $x_{0}, y_{0}$ and $\sigma$ are all in degrees of visual angle.

Next, we define the effective stimulus, $s(x, y, t)$. In the experiments reported here, the stimulus contrast pattern, either a checkerboard or a dartboard, is revealed as the stimulus aperture moves across the pattern. We assume that all parts of the pattern within the aperture contribute equally to the fMRI response. This assumption is justified because fMRI responses to these kinds of stimuli are dominated by contrast edges and the checks within the aperture are moving so that contrast edges are present throughout the aperture (Engel et al., 1997). With these stimuli and apertures, the formula describing the effective stimulus is simply a binary indicator function that marks the position of the stimulus aperture at each time. 
For a given pRF model and effective stimulus, we calculate the predicted pRF response. Because the $\mathrm{pRF}$ and effective stimulus formula are defined in the common units of visual space, the first step towards predicting the fMRI time series is to calculate the overlap between the effective stimulus and the model $\mathrm{pRF}$ at a voxel

$$
r(t)=\sum_{x, y} s(x, y, t) g(x, y)
$$

We then obtained the time series prediction by convolving $r(t)$ with a model of the hemodynamic response function (HRF; $h(t)$ ) (Boynton et al., 1996; Friston et al., 1998). We estimated $h(t)$ separately for each subject (Appendix A).

$$
p(t)=r(t) * h(t)
$$

The goodness-of-fit is estimated by computing the residual sum of squares (RSS) between the prediction, $p(t)$, and the data, $y(t)$. We calculate this error term allowing for a scale factor, $\beta$, that accounts for the unknown units of the fMRI signal

$$
\mathrm{RSS}=\sum_{t}(y(t)-p(t) \beta)^{2}
$$

The optimal pRF parameters were found by minimizing the RSS using a two-stage coarseto-fine search. To do this, the fMRI data were resampled to an isotropic 1-mm resolution within the identified gray matter of the cortex. In the first stage, the fMRI data were smoothed along the cortical surface using a diffusion smoothing process that approximated a 5-mm full-width at half-maximum Gaussian kernel (Andrade et al., 2001). Hence, the smoothing process respects the topology of the cortical surface.

We then generated 100,000 different fMRI time series predictions by varying the pRF model parameters, $x_{0}, y_{0}$ and $\sigma$, across a wide range of plausible values on a regular sampling grid. The spatial smoothing increases the signal-to-noise ratios by removing high spatial frequency noise, and it imposes a spatial correlation between voxels. Hence, we can safely estimate the parameters on a sub-sample of the voxels (every other voxel) and accurately interpolate the remaining voxels. Hence, the smoothing provides a good first estimate of the pRF parameters in minimal processing time. Importantly, the grid search is guaranteed to find a global minimum at the coarse grid sampling density.

In the second stage, we apply an optimization algorithm (Fletcher and Powell, 1963) to the 1-mm data for every voxel whose first stage estimates explain more than $15 \%$ of the variance of the coarse fMRI signal. In the second stage, no smoothing is applied; the final pRF size estimates are derived by fitting data without any of the spatial smoothing used in the first stage. The coarse-to-fine approach minimizes the processing time and increases the likelihood of finding a global minimum.

We estimate three pRF model parameters for each voxel independently: $x_{0}, y_{0}$ and $\sigma$. These are estimated simultaneously using multiple fMRI time series measured with several different stimulus apertures (rings, wedges, and bars). In the experiments described here, each stimulus cycle comprises 16 time points and each cycle is repeated six times per scan. 
We use up to six different stimulus apertures (4 bars, rings, and wedges). Hence, the three pRF parameters are estimated from a total of 96 measured values, repeated roughly 36 times.

Various descriptions of the data can be derived from the pRF fit, including traditional eccentricity and polar-angle maps. Importantly, the Gaussian width parameter is a novel estimate that provides information about the population receptive field size. We can also derive the percent variance explained and conventional statistical $t$ - and $p$-maps that specify how well the pRF linear model fits the time series. We note that the $t$-values (and $p$-values) are biased towards larger (smaller) values because they result from the best fits out of a large search space. Therefore we use percent variance explained as our primary measure of the goodness-of-fit.

\section{Results}

\section{V1 and LO respond differently to identical traveling wave stimuli}

The V1 and lateral occipital (LO) responses to the same stimulus differ qualitatively. The time series in Figs. 3A and B are typical examples of how the responses to a rotating wedge stimulus differ. These voxels respond preferentially to a similar wedge position, conventional analyses would assign them similar phases (Engel et al., 1994; Sereno et al., $1995)$, and duty-cycles or duty-cycle-related measures (21.5\% and 33.5\%, respectively) (Larsson and Heeger, 2006; Li et al., 2007; Smith et al., 2001). However, the V1 modulation is much higher than the LO modulation (Figs. $3 \mathrm{C}$ and D). The low response modulation to the rotating wedge stimulus in LO cortex is typical, and the same low modulation is found in ventral occipital (VO). Some groups concluded that the low modulation in these regions implies that there are no angular maps in LO or VO (Levy et al., 2001; Tootell et al., 1996; Tootell and Hadjikhani, 2001). Other groups find small but reliable modulations and maps in these regions (Brewer et al., 2005; Larsson and Heeger, 2006; Wandell et al., 2005).

\section{The responses differences are explained by $\mathrm{pRF}$ size differences}

Why are the LO response modulations much smaller than those in V1? The reason is clarified by measurements using a stimulus containing the mean-luminance (zero-contrast) blocks (Fig. 1F). Inserting the mean-luminance block alters the time series in V1 only when the mean-luminance replaces a wedge at the preferred position (Fig. 3E). The LO response, however, drops whenever the mean-luminance block is inserted (Fig. 3F). We conclude that the V1 voxel responds only to a narrow range of wedge positions; the LO voxel responds to some extent to all wedge positions.

These differences are captured by the pRF model in the population receptive field parameters. These are derived from rotating wedge (Figs. 4A and B) and expanding ring (Figs. 4C and D) responses to stimuli that include mean-luminance blocks. The pRF estimates for the voxel in V1 and LO are shown Figs. 4E and F.

The $\mathrm{pRF}$ center position in visual space, $\left(x_{0}, y_{0}\right)$, is similar for the $\mathrm{V} 1$ and $\mathrm{LO}$ voxels. The differences between the time series are explained entirely by the larger pRF size $(\sigma)$ in LO compared to V1. The pRF model predicts the V1 and LO time-series about equally well. Thus, the reduced modulation in LO in conventional mapping experiments can be explained by the difference in pRF size.

The difficulty in measuring LO and VO maps with conventional ring and wedge stimuli arises because large receptive fields are poorly analyzed by these stimuli. Inserting a meanluminance block provides an important baseline condition. Without this baseline these large differences in pRF size cannot be distinguished. As we illustrate next, improving the 
quantitative analysis will enable us to measure maps and pRF properties in a much larger extent of cortex, including LO and VO.

\section{The model-based approach measures more accurate visual field maps than conventional approaches}

The pRF center positions define a visual field map. Next, we compare the pRF visual field maps estimates with those derived using conventional phase-encoding method (Engel et al., 1994).

Specifically, we compute a correlation coefficient that measures the agreement between visual field maps estimated from the same data using the two methods (Dumoulin et al., 2003). The correlation coefficient is computed using all voxels within the cortex with a response modulation whose coherence exceeds 0.3 ( $p<0.003$, uncorrected) (Bandettini et al., 1993; Brewer et al., 2005). The position estimates of the phase-encoded and pRF modelbased method are highly correlated (Table 1 , all correlations are significant $p<0.0001$ ). The correlation levels are similar for maps derived using conventional stimuli or stimuli with mean-luminance blocks inserted.

The correlation coefficients between methods are consistently higher for angular than eccentricity estimates. This is because the conventional phase-encoding eccentricity estimate is slightly distorted. This distortion can be traced to an interaction between the ring stimuli and pRF size. In Appendix B, we explain this interaction and show that the pRF modelbased method is more accurate than the phase-encoding method.

An important advantage of the pRF model-based method is that visual field maps can be derived from responses to a wide array of stimuli, including those that are not well-suited to the phase-encoding method. To evaluate the consistence of these estimates, we correlated the V1-V3 field maps derived using different stimuli (Figs. 1E-G), including stimuli that conventional phase-encoded methods cannot analyze (Fig. 1G). The average correlation coefficient $(r \pm$ S.D.) for the maps estimated using the three types of stimuli was $0.84 \pm 0.06$ (three subjects). The high correlation is similar to the correlation observed between two sessions when using the same stimuli $(0.88 \pm 0.02)$ and when measuring conventional visual field sign maps $(0.79 \pm 0.06)$ (Dumoulin et al., 2003). Hence, the pRF visual field maps are stimulus-independent but the exact stimulus parameters will determine the precision of the estimates (Wandell et al., 2005).

The pRF visual field maps derived by combining the measurements with bars, rings and wedges (including mean-luminance blocks) are shown as angular and eccentricity maps in Fig. 5. The maps are shown on an inflated cortical surface in the region near the occipital pole (Fig. 5A). The stimuli covered only the central $3^{\circ}$ radius of the visual field. For many years, labs using conventional methods stopped visual field mapping at $0.5^{\circ}-2^{\circ}$ eccentricity, believing that central foveal maps could not be accurately measured (Dougherty et al., 2003;Liu et al., 2005;Schira et al., 2007;Silver et al., 2007;Somers et al., 1999;Tootell and Hadjikhani, 2001). Notice that with the pRF model-based method, we trace with high precision the visual field maps to the center of the foveal representation. For example, we can find boundaries between V1, V2 and V3 at an eccentric representation below half a degree (Figs. 5B and C).

\section{Population receptive field size is $5 \times$ larger in LO and VO than V1-V3}

Fitting a symmetric Gaussian pRF model produces both visual field map and $\mathrm{pRF}$ size estimates $(\sigma)$. The $\mathrm{pRF}$ size estimates from a $14^{\circ}$ radius field of view are shown for three subjects in Fig. 6. There is a significant increase in pRF size as one compares the responses in V1-V3 $\left(\sigma \sim 0.5^{\circ}-2^{\circ}\right)$ with those in $\mathrm{LO}$ and $\mathrm{VO}\left(\sigma \sim 4^{\circ}-8^{\circ}\right)$. 
The quality of the pRF fit to the data is illustrated by comparing the measured and predicted time series in Fig. 4. For all of the data shown in Fig. 6 (and Supplementary Fig. 1) the average (minimum) percent variance-accounted-for $\left(r^{2}\right)$ is $60 \%(25 \%)$.

\section{The $\mathrm{pRF}$ is lateralized in V1-V3 but not in LO and VO}

The laterality of the pRFs varies across cortex. The pseudo-color overlay in Fig. 7 indicates the percentage of the pRF that is in the ipsilateral visual field. In V1-V3, the pRFs are confined largely to the contralateral visual field; the main exception occurs at the vertical meridian representations that separate V1/V2 and the vertical meridian of V3. At these boundaries, the pRF extends into the ipsilateral visual field (Fig. 7).

The pRFs in LO and VO extend significantly into the ipsilateral visual field. These laterality estimates quantify previous observations of ipsilateral responses in LO (Hemond et al., 2007; Niemeier et al., 2005; Tootell et al., 1998). We note that the pRF estimates here are based on a circularly symmetric Gaussian model; it may be that models allowing asymmetric shapes will result in a different assessment of laterality.

\section{Population receptive field size estimates increase with eccentricity in V1-V3}

Within V1-V3, the pRF size increases as a function of eccentricity (Figs. 8A-C). The pRFsizes increase systematically; the within-map increase is roughly twofold when measuring from $1^{\circ}$ to $12^{\circ}$ of eccentricity. The size increases by about a factor of 4 between V1 and V3, and a factor of five or six between V1 and LO.

\section{Discussion}

We introduce a functional MRI method that computes a model of the pRF from responses to a wide range of stimuli. The method estimates both a visual field map estimate as well as other neuronal population properties, including size and laterality. We show that the visual field maps are more accurate than conventional methods. The eccentricity maps are improved by the new method, whereas the polar-angle maps around the fovea are improved by the new methods in combination with new stimuli. We report quantitative estimates of pRF size in medial, lateral and ventral occipital regions of human visual cortex. We show that incorporating mean-luminance baseline stimulus conditions is essential to estimate the pRF size. Also, we quantify the amount of input from ipsi- and contralateral visual fields. The human pRF size vary across visual field maps as well as within a visual field maps as a function of eccentricity. The method is non-invasive and can be applied to a wide range of conditions when it is useful to link fMRI signals to models of the neuronal population.

\section{Comparisons of human pRF and neuronal receptive fields}

We compare the human pRF size estimates with independent pRF estimates made using single- and multi-unit activity (S/MUA) and local field potentials (LFP) in non-human primates (Fig. 9). We make these comparisons for pRF size measurements in V1 (Gattass et al., 1981;Gattass et al., 1987;Van Essen et al., 1984;Victor et al., 1994), V2 (Burkhalter and Van Essen, 1986;Gattass et al., 1981;Rosa et al., 1988) and V3/VP (Burkhalter et al., 1986; Felleman and Van Essen, 1987;Newsome et al., 1986;Rosa et al., 2000).

This comparison links electrophysiological and fMRI pRF estimates but the comparisons are limited by differences in experimental procedures, such as stimuli, species, anesthetics and pRF size criteria. The electrophysiological studies generally measured the neuronal-pRF area qualitatively. To make a quantitative comparison with the reports from the literature, we assumed the electrophysiological measurements correspond to the full area at half 
maximum (Victor et al., 1994). In the future, it will be possible to improve the quantification in the animal experiments.

There are many similar trends in the two sets of measurements, but the fMRI estimates are generally larger than the S/MUA estimates. This is expected because the fMRI signal samples a larger neural population than the S/MUA measurements. The larger sample has wider variance in visual field position, and this should increase the human pRF size estimate (see discussion near Eq. (6)). The fMRI-pRF estimates are very close to the LFP-pRF estimate, which is expected since the fMRI signal is correlated more with LFP than S/MUA signals (Logothetis et al., 2001). Within each visual field map, the rate of increase the visual field eccentricity follows a similar slope.

In addition, the fMRI-pRF sizes are comparable to human electrophysiological measurements estimated using surface electrode with a 2.2-mm-diameter recording area (Yoshor et al., 2007). Yoshor et al. (2007) report pRF sizes in early visual cortex, roughly corresponding to $\mathrm{V} 1 / \mathrm{V} 2$, as $\sigma=0.76^{\circ} \pm 0.23^{\circ}$. Sizes in later visual cortex roughly corresponding to $\mathrm{V} 3 / \mathrm{V} 3 \mathrm{~A} / \mathrm{hV} 4$, are estimated as $\sigma=1.49^{\circ} \pm 0.81^{\circ}$. Both of these values are consistent with the fMRI-pRF size estimates.

\section{Nuisance and HRF factors influence pRF estimates}

The estimated pRF size depends on a number of unwanted factors; only some of these are explicitly modeled. These include eye-movements, head-movement, brain pulsatility and optical defocus. All of these factors create a bias towards larger estimated pRF size; they add noise, but no bias, to the visual field position estimates.

The temporal hemodynamic response function (HRF) is the most important non-neural influence on the pRF size estimate. Differences between the true and assumed HRF also influence estimated pRF parameters. Given its importance, we explicitly model the temporal HRF and derive subject-specific fits (Appendix A). We model the HRF as a space-time invariant convolution kernel (Boynton et al., 1996; Logothetis and Wandell, 2004; Worsley et al., 2002) and we show that inexact characterization of the HRF temporal spread influences the absolute values of our pRF measurements, but not relative pRF measurements (Appendix C).

We used a space-invariant HRF because of its relative simplicity and because it is possible to obtain a secure estimate of the HRF by averaging over cortex. It may be, however, that the HRF varies across cortex within individuals (Handwerker et al., 2004; Miezin et al., 2000). Systematic failures of HRF space-invariance may introduce systematic errors into the pRF size estimate. In particular for our stimulus design, increased HRF widths would be interpreted as increased pRF sizes. There are several reasons why this possibility is unlikely to explain any of the key results from our measurements.

First, the BOLD response to the same stimulus in V1 and LO (see Figs. 3E and F) differ enormously. The difference is more 10 times larger than the typical HRF variation and therefore the difference between these responses is not due to HRF variation (Aguirre et al., 1998; de Zwart et al., 2005; Handwerker et al., 2004; Miezin et al., 2000; Neumann et al., 2003).

Second, the small variations within cortical maps, such as the changes we observe between central and peripheral representations within $\mathrm{V} 1-\mathrm{V} 3$, are consistent with neuronal properties (Fig. 9). 
Third, we made additional measurements of the spatial HRF variation to see whether the differences might predict estimated pRF size (Fig. 10). Specifically, we estimated the HRF a full-width at half-maximum of a canonical model of the HRF at every cortical location. We then plotted the estimated pRF size as a function of the estimated HRF width. There is a weak negative correlation $(r=-0.1)$ between the HRF width and the pRF size (Fig. 10; Supplementary Fig. 2). Hence, the pRF size differences are not explained by increasing HRF widths. We repeated this analysis using two different models of the HRF and the results are the same for both models (Boynton et al., 1996;Friston et al., 1998).

The spatial-HRF also can influence the pRF parameters. Experimental evidence does not reveal systematic variation in the spatial spread of the HRF (Das and Gilbert, 1995; Engel et al., 1997). While these are only limited measurements, the reported variance in the spatial extent of the HRF $(<1 \mathrm{~mm})$ is unlikely to play a significant role in the fMRI data given the effective resolution of the fMRI voxels $\left(2.5 \times 2.5 \times 3 \mathrm{~mm}^{3}\right)$. In the future, with more precise estimates of the HRF across cortex, it should be possible to extend the HRF analysis to include the spatial component (3-4 mm full-width at half-maximum along the cortical surface (Das and Gilbert, 1995; Engel et al., 1997; Iadecola et al., 1997; Malonek and Grinvald, 1996; Shmuel et al., 2007)). By assuming no spatial spread, we introduce a bias towards increased $\mathrm{pRF}$ size.

\section{Neuronal factors}

The pRF is a statistical summary of the neuronal properties within the sampled region, and pRF model parameters depend on the size and intrinsic properties of the sampled neuronal population. In many ways, the sampling problems in fMRI parallel those in electrophysiology. In the case of electrophysiology, measurement parameters such as electrode impedance determine the neuronal sampling properties and membrane properties of certain neurons may produce larger amplitude action potentials (Logothetis, 2003). In fMRI, the voxel size influences the neuronal sampling population and the metabolic influence of certain neurons may be greater. In both cases, certain stimuli but not others may drive neuronal responses, further biasing the sampling of the neural population.

There are three main neuronal factors that contribute to the pRF size estimate. First, the pRF is dependent on the average receptive field of the neurons that drive the response. This includes both the classical receptive field as well as any responses driven by stimuli beyond the classical receptive field. Second, the pRF is influenced by the position scatter of the individual receptive fields. The neuronal receptive field and scatter have been reported to covary (Hubel and Wiesel, 1974). Third, the fMRI response from a cortical voxel may be driven by different groups of cells depending on the stimulus properties.

Suppose we consider only a single type of stimulus and assume that signals are from a single neuronal population. The pRF size estimate depends on a combination of neural and nonneural components.

$$
\sigma_{\mathrm{pRF}}^{2}=\sigma_{\mathrm{nRF}}^{2}+\sigma_{\text {position }}^{2}+k
$$

where $\sigma_{\mathrm{nRF}}$ is the mean size of the neuronal receptive field, $\sigma_{\text {position }}$ is the position variance, and $k$ is a constant factor that captures the non-neural factors.

This formal description of the pRF size should enable us, ultimately, to separate different factors and improve the estimates of individual neuronal properties. For example, if we have some indication of the position variance, we can account for this in the modeling. Similarly, 
additional information about the spatial spread of the HRF or other non-neural factors will enable us to sharpen our estimates.

We distinguish two stimulus components: the carrier and the aperture. The carrier is the background pattern that predominantly drives neuronal activity. In this study, the carrier is a checkerboard pattern. The aperture reveals the carrier. In this study, the aperture is a wedge, ring or bar. The pRF model predicts the fMRI response using the position of the aperture (see Fig. 2) as an independent variable and assigns the carrier as a side-condition.

As we change the carrier, we expect that to stimulate different neuronal populations. In some cases, the change in neuronal population will cause the pRF model estimates to change. For example, we expect pRF sizes to vary as we change properties of the carrier, such as its color, contrast, and motion. The pRF model-based method can be used to verify that certain carriers predominantly stimulate pathways (magnocellular, parvocellular, and koniocellular) that are known to have different receptive field sizes (Rodieck, 1998).

\section{Future extensions and applications}

In the analyses here, we use a single pRF model: an isotropic 2D Gaussian. This model provides a compact description of the pRF using only three parameters. As we collect more data, it will become possible to compare more complex pRF models with significant statistical power. For example, the pRF can be modeled as an anisotropic Gaussian to test hypotheses concerning integration biases in the pRF as suggested by some studies (Sasaki et al., 2006).

Additionally, the pRF may be modeled using nonlinear models and sums and differences of Gaussians to test hypotheses about surround suppression. These suppressive effects have been demonstrated using fMRI (Dumoulin and Hess, 2006; Kastner et al., 2001; Williams et al., 2003; Zenger-Landolt and Heeger, 2003), and it has been shown that the strength and spatial extent of these suppressive effects increase in later visual areas (Kastner et al., 2001; Zenger-Landolt and Heeger, 2003). This increase may be related to increases in the RF sizes in these areas (Bles et al., 2006; Kastner et al., 2001).

Furthermore, we note that some investigators include visual field maps and 2D Gaussian pRF models in algorithms for inferring the stimulus from the fMRI response (Thirion et al., 2006). Preliminary work on this topic has incorporated a constant pRF size $\left(\sigma=0.75^{\circ}\right)$. We suspect that better estimates of the pRF properties from the data should improve algorithms in that field. The large increase in pRF size in LO (Fig. 6) and the decrease in laterality (Fig. 7) support the hypothesis that these regions perform functions distinct from other map clusters (Grill-Spector et al., 2001; Malach et al., 1995; Wandell et al., 2005). In future experiments, we will be able to combine detailed mapping and pRF size estimates to further clarify whether these changes in pRF properties are tightly coupled to the transitions between maps and clusters.

The properties of the neuronal-pRF may change during development or as a consequence of various disease conditions. In this case, the fMRI measurements may be of value in early diagnosis or to track healthy development.

\section{Conclusion}

The pRF method links fMRI measurements at the millimeter scale to response neuronal properties at the micron scale and reduces the gap between functional imaging and electrophysiology. 
The estimated human pRF agrees with electrophysiological and conventional fMRI estimates in three ways. First, the pRF-derived visual field maps agree well with those estimated from monkey electrophysiology and conventional human fMRI visual field mapping methods (Fig. 5). Second, pRF size increases systematically from V1 to V3 (Fig. 8), matching electrophysiological measurements (Fig. 9). Third, pRF size estimates across the visual cortex agree with estimates acquired using cortical surface electrodes in humans (Yoshor et al., 2007).

\section{Supplementary Material}

Refer to Web version on PubMed Central for supplementary material.

\section{Acknowledgments}

This work was supported by a National Eye Institute Grant RO1 EY03164 to BW and a Larry L. Hillblom Foundation fellowship 2005/2BB to SD. We thank Kaoru Amano, Michal Ben-Shachar, Alyssa Brewer, SingHuang Cheung, Robert Dougherty, Kalanit Grill-Spector, Yoichiro Masuda, and Rory Sayres for their help and comments on the manuscript. Parts of this work have been published in abstract form (Dumoulin et al., 2006). The software is freely available and is distributed as part of the VISTA software (http://white.stanford.edu/software/).

\section{References}

Aguirre GK, Zarahn E, D'Esposito M. The variability of human, BOLD hemodynamic responses. NeuroImage. 1998; 8:360-369. [PubMed: 9811554]

Andrade A, Kherif F, Mangin JF, Worsley KJ, Paradis AL, Simon O, Dehaene S, Le Bihan D, Poline JB. Detection of fMRI activation using cortical surface mapping. Hum Brain Mapp. 2001; 12:7993. [PubMed: 11169872]

Ashburner, J.; Friston, KJ. Rigid body registration. In: Penny, WD., editor. Human Brain Function. Academic Press; 2003.

Bandettini PA, Jesmanowicz A, Wong EC, Hyde JS. Processing strategies for time-course data sets in functional MRI of the human brain. Magn Reson Med. 1993; 30:161-173. [PubMed: 8366797]

Birn RM, Saad ZS, Bandettini PA. Spatial heterogeneity of the nonlinear dynamics in the fMRI BOLD response. NeuroImage. 2001; 14:817-826. [PubMed: 11554800]

Bles M, Schwarzbach J, De Weerd P, Goebel R, Jansma BM. Receptive field size-dependent attention effects in simultaneously presented stimulus displays. NeuroImage. 2006; 30:506-511. [PubMed: 16256374]

Boynton GM, Engel SA, Glover GH, Heeger DJ. Linear systems analysis of functional magnetic resonance imaging in human V1. J Neurosci. 1996; 16:4207-4221. [PubMed: 8753882]

Brainard DH. The psychophysics toolbox. Spat Vis. 1997; 10:433-436. [PubMed: 9176952]

Brewer AA, Liu J, Wade AR, Wandell BA. Visual field maps and stimulus selectivity in human ventral occipital cortex. Nat Neurosci. 2005; 8:1102-1109. [PubMed: 16025108]

Burkhalter A, Van Essen DC. Processing of color, form and disparity information in visual areas VP and V2 of ventral extrastriate cortex in the macaque monkey. J Neurosci. 1986; 6:2327-2351. [PubMed: 3746412]

Burkhalter A, Felleman DJ, Newsome WT, Van Essen DC. Anatomical and physiological asymmetries related to visual areas V3 and VP in macaque extrastriate cortex. Vision Res. 1986; 26:63-80. [PubMed: 3716214]

Das A, Gilbert CD. Long-range horizontal connections and their role in cortical reorganization revealed by optical recording of cat primary visual cortex. Nature. 1995; 375:780-784. [PubMed: 7596409]

DeYoe EA, Carman GJ, Bandettini P, Glickman S, Wieser J, Cox R, Miller D, Neitz J. Mapping striate and extrastriate visual areas in human cerebral cortex. Proc Natl Acad Sci U S A. 1996; 93:23822386. [PubMed: 8637882] 
de Zwart JA, Silva AC, van Gelderen P, Kellman P, Fukunaga M, Chu R, Koretsky AP, Frank JA, Duyn JH. Temporal dynamics of the BOLD fMRI impulse response. NeuroImage. 2005; 24:667677. [PubMed: 15652302]

Dougherty RF, Koch VM, Brewer AA, Fischer B, Modersitzki J, Wandell BA. Visual field representations and locations of visual areas V1/2/3 in human visual cortex. J Vis. 2003; 3:586598. [PubMed: 14640882]

Dumoulin SO, Hess RF. Modulation of V1 activity by shape: image-statistics or shape-based perception? J. Neurophysiol. 2006; 95:3654-3664.

Dumoulin SO, Hoge RD, Baker CL Jr, Hess RF, Achtman RL, Evans AC. Automatic volumetric segmentation of human visual retinotopic cortex. NeuroImage. 2003; 18:576-587. [PubMed: 12667835]

Dumoulin SO, Brewer AA, Ben-Shachar M, Dougherty RF, Wandell BA. Distinguishing visual field map clusters: a new paradigm. J Vis. 2006; 6:533-533.

Engel SA, Rumelhart DE, Wandell BA, Lee AT, Glover GH, Chichilnisky EJ, Shadlen MN. fMRI of human visual cortex. Nature. 1994; 369:525. [PubMed: 8031403]

Engel SA, Glover GH, Wandell BA. Retinotopic organization in human visual cortex and the spatial precision of functional MRI. Cereb Cortex. 1997; 7:181-192. [PubMed: 9087826]

Felleman DJ, Van Essen DC. Receptive field properties of neurons in area V3 of macaque monkey extrastriate cortex. J Neurophysiol. 1987; 57:889-920. [PubMed: 3585463]

Fletcher R, Powell MJD. A rapidly convergent descent method for minimization. Comput J. 1963; 6:163-168.

Friston KJ, Holmes AP, Poline JB, Grasby PJ, Williams SC, Frackowiak RS, Turner R. Analysis of fMRI time-series revisited. NeuroImage. 1995; 2:45-53. [PubMed: 9343589]

Friston KJ, Fletcher P, Josephs O, Holmes A, Rugg MD, Turner R. Event-related fMRI: characterizing differential responses. Neuro-Image. 1998; 7:30-40. [PubMed: 9500830]

Gattass R, Gross CG, Sandell JH. Visual topography of V2 in the macaque. J Comp Neurol. 1981; 201:519-539. [PubMed: 7287933]

Gattass R, Sousa AP, Rosa MG. Visual topography of V1 in the Cebus monkey. J Comp Neurol. 1987; 259:529-548. [PubMed: 3597827]

Glover GH. Deconvolution of impulse response in event-related BOLD fMRI. NeuroImage. 1999a; 9:416-429. [PubMed: 10191170]

Glover GH. Simple analytic spiral K-space algorithm. Magn Reson Med. 1999b; 42:412-415. [PubMed: 10440968]

Glover GH, Lai S. Self-navigated spiral fMRI: interleaved versus single-shot. Magn Reson Med. 1998; 39:361-368. [PubMed: 9498591]

Grill-Spector K, Kourtzi Z, Kanwisher N. The lateral occipital complex and its role in object recognition. Vision Res. 2001; 41:1409-1422. [PubMed: 11322983]

Handwerker DA, Ollinger JM, D'Esposito M. Variation of BOLD hemodynamic responses across subjects and brain regions and their effects on statistical analyses. NeuroImage. 2004; 21:16391651. [PubMed: 15050587]

Hansen KA, David SV, Gallant JL. Parametric reverse correlation reveals spatial linearity of retinotopic human V1 BOLD response. NeuroImage. 2004; 23:233-241. [PubMed: 15325370]

Hemond CC, Kanwisher NG, Op de Beeck HP. A preference for contralateral stimuli in human objectand face-selective cortex. PLoS ONE. 2007; 2:e574. [PubMed: 17593973]

Hubel DH, Wiesel TN. Uniformity of monkey striate cortex: a parallel relationship between field size, scatter, and magnification factor. J Comp Neurol. 1974; 158:295-305. [PubMed: 4436457]

Iadecola C, Yang G, Ebner TJ, Chen G. Local and propagated vascular responses evoked by focal synaptic activity in cerebellar cortex. J Neurophysiol. 1997; 78:651-659. [PubMed: 9307102]

Kastner S, De Weerd P, Pinsk MA, Elizondo MI, Desimone R, Ungerleider LG. Modulation of sensory suppression: implications for receptive field sizes in the human visual cortex. $\mathrm{J}$ Neurophysiol. 2001; 86:1398-1411. [PubMed: 11535686]

Lange N, Zeger SL. Non-linear Fourier time series analysis for human brain mapping by functional magnetic resonance imaging. J R Stat Soc, Ser C, Appl Stat. 1997; 46:1-29. 
Larsson J, Heeger DJ. Two retinotopic visual areas in human lateral occipital cortex. J Neurosci. 2006; 26:13128-13142. [PubMed: 17182764]

Levy I, Hasson U, Avidan G, Hendler T, Malach R. Center-periphery organization of human object areas. Nat Neurosci. 2001; 4:533-539. [PubMed: 11319563]

Li X, Dumoulin SO, Mansouri B, Hess RF. The fidelity of the cortical retinotopic map in human amblyopia. Eur J Neurosci. 2007; 25:1265-1277. [PubMed: 17425555]

Liu T, Pestilli F, Carrasco M. Transient attention enhances perceptual performance and fMRI response in human visual cortex. Neuron. 2005; 45:469-477. [PubMed: 15694332]

Logothetis NK. The underpinnings of the BOLD functional magnetic resonance imaging signal. J Neurosci. 2003; 23:3963-3971. [PubMed: 12764080]

Logothetis NK, Wandell BA. Interpreting the BOLD signal. Annu Rev Physiol. 2004; 66:735-769. [PubMed: 14977420]

Logothetis NK, Pauls J, Augath M, Trinath T, Oeltermann A. Neurophysiological investigation of the basis of the fMRI signal. Nature. 2001; 412:150-157. [PubMed: 11449264]

Maes F, Collignon A, Vandermeulen D, Marchal G, Suetens P. Multimodality image registration by maximization of mutual information. IEEE Trans Med Imag. 1997; 16:187-198.

Malach R, Reppas JB, Benson RR, Kwong KK, Jiang H, Kennedy WA, Ledden PJ, Brady TJ, Rosen $\mathrm{BR}$, Tootell RB. Object-related activity revealed by functional magnetic resonance imaging in human occipital cortex. Proc Natl Acad Sci U S A. 1995; 92:8135-8139. [PubMed: 7667258]

Malonek D, Grinvald A. Interactions between electrical activity and cortical microcirculation revealed by imaging spectroscopy: implications for functional brain mapping. Science. 1996; 272:551-554. [PubMed: 8614805]

Miezin FM, Maccotta L, Ollinger JM, Petersen SE, Buckner RL. Characterizing the hemodynamic response: effects of presentation rate, sampling procedure, and the possibility of ordering brain activity based on relative timing. Neuroimage. 2000; 11:735-759. [PubMed: 10860799]

Nestares O, Heeger DJ. Robust multiresolution alignment of MRI brain volumes. Magn Reson Med. 2000; 43:705-715. [PubMed: 10800036]

Neumann J, Lohmann G, Zysset S, von Cramon DY. Within-subject variability of BOLD response dynamics. NeuroImage. 2003; 19:784-796. [PubMed: 12880807]

Newsome WT, Maunsell JH, Van Essen DC. Ventral posterior visual area of the macaque: visual topography and areal boundaries. J Comp Neurol. 1986; 252:139-153. [PubMed: 3782504]

Niemeier M, Goltz HC, Kuchinad A, Tweed DB, Vilis T. A contralateral preference in the lateral occipital area: sensory and attentional mechanisms. Cereb Cortex. 2005; 15:325-331. [PubMed: 15269109]

Pelli DG. The VideoToolbox software for visual psychophysics: transforming numbers into movies. Spat Vis. 1997; 10:437-442. [PubMed: 9176953]

Rodieck, RW. The First Steps in Seeing. Sinauer Associates, Inc; Sunderland, MA: 1998.

Rosa MG, Sousa AP, Gattass R. Representation of the visual field in the second visual area in the Cebus monkey. J Comp Neurol. 1988; 275:326-345. [PubMed: 3225342]

Rosa MG, Pinon MC, Gattass R, Sousa AP. “Third tier” ventral extrastriate cortex in the New World monkey, Cebus apella. Exp Brain Res. 2000; 132:287-305. [PubMed: 10883378]

Sasaki Y, Rajimehr R, Kim BW, Ekstrom LB, Vanduffel W, Tootell RB. The radial bias: a different slant on visual orientation sensitivity in human and nonhuman primates. Neuron. 2006; 51:661670. [PubMed: 16950163]

Schira MM, Wade AR, Tyler CW. Two-dimensional mapping of the central and parafoveal visual field to human visual cortex. J Neurophysiol. 2007; 97:4284-4295. [PubMed: 17360817]

Sereno MI, Dale AM, Reppas JB, Kwong KK, Belliveau JW, Brady TJ, Rosen BR, Tootell RB. Borders of multiple visual areas in humans revealed by functional magnetic resonance imaging. Science. 1995; 268:889-893. [PubMed: 7754376]

Shmuel A, Yacoub E, Chaimow D, Logothetis NK, Ugurbil K. Spatio-temporal point-spread function of fMRI signal in human gray matter at 7 Tesla. NeuroImage. 2007; 35:539-552. [PubMed: 17306989] 
Silver MA, Ress D, Heeger DJ. Neural correlates of sustained spatial attention in human early visual cortex. J Neurophysiol. 2007; 97:229-237. [PubMed: 16971677]

Smith AT, Singh KD, Williams AL, Greenlee MW. Estimating receptive field size from fMRI data in human striate and extrastriate visual cortex. Cereb Cortex. 2001; 11:1182-1190. [PubMed: 11709489]

Somers DC, Dale AM, Seiffert AE, Tootell RB. Functional MRI reveals spatially specific attentional modulation in human primary visual cortex. Proc Natl Acad Sci U S A. 1999; 96:1663-1668. [PubMed: 9990081]

Teo PC, Sapiro G, Wandell BA. Creating connected representations of cortical gray matter for functional MRI visualization. IEEE Trans Med Imag. 1997; 16:852-863.

Thirion B, Duchesnay E, Hubbard E, Dubois J, Poline JB, Lebihan D, Dehaene S. Inverse retinotopy: inferring the visual content of images from brain activation patterns. NeuroImage. 2006; 33:11041116. [PubMed: 17029988]

Tootell RB, Hadjikhani N. Where is 'dorsal V4' in human visual cortex? Retinotopic, topographic and functional evidence. Cereb Cortex. 2001; 11:298-311. [PubMed: 11278193]

Tootell RB, Dale AM, Sereno MI, Malach R. New images from human visual cortex. Trends Neurosci. 1996; 19:481-489. [PubMed: 8931274]

Tootell RB, Mendola JD, Hadjikhani NK, Ledden PJ, Liu AK, Reppas JB, Sereno MI, Dale AM. Functional analysis of V3A and related areas in human visual cortex. J Neurosci. 1997; 17:70607078. [PubMed: 9278542]

Tootell RB, Mendola JD, Hadjikhani NK, Liu AK, Dale AM. The representation of the ipsilateral visual field in human cerebral cortex. Proc Natl Acad Sci U S A. 1998; 95:818-824. [PubMed: 9448246]

Van Essen DC, Newsome WT, Maunsell JH. The visual field representation in striate cortex of the macaque monkey: asymmetries, anisotropies, and individual variability. Vision Res. 1984; 24:429-448. [PubMed: 6740964]

Victor JD, Purpura K, Katz E, Mao B. Population encoding of spatial frequency, orientation, and color in macaque V1. J Neurophysiol. 1994; 72:2151-2166. [PubMed: 7884450]

Wandell BA, Chial S, Backus BT. Visualization and measurement of the cortical surface. J Cogn Neurosci. 2000; 12:739-752. [PubMed: 11054917]

Wandell BA, Brewer AA, Dougherty RF. Visual field map clusters in human cortex. Philos Trans R Soc Lond, B Biol Sci. 2005; 360:693-707. [PubMed: 15937008]

Williams AL, Singh KD, Smith AT. Surround modulation measured with functional MRI in the human visual cortex. J Neurophysiol. 2003; 89:525-533. [PubMed: 12522199]

Worsley KJ, Friston KJ. Analysis of fMRI time-series revisited-Again. NeuroImage. 1995; 2:173181. [PubMed: 9343600]

Worsley KJ, Liao CH, Aston J, Petre V, Duncan GH, Morales F, Evans AC. A general statistical analysis for fMRI data. NeuroImage. 2002; 15:1-15. [PubMed: 11771969]

Yoshor D, Bosking WH, Ghose GM, Maunsell JH. Receptive Fields in Human Visual Cortex Mapped with Surface Electrodes. Cereb Cortex. 2007; 17 (10):2293-2302. [PubMed: 17172632]

Zenger-Landolt B, Heeger DJ. Response suppression in V1 agrees with psychophysics of surround masking. J Neurosci. 2003; 23:6884-6893. [PubMed: 12890783]

\section{Appendix A. HRF estimation}

The HRF was derived from V1 responses to flickering checkerboards (Fig. 11). The responses were measured in the region that represents between $5^{\circ}$ and $9^{\circ}$ of visual eccentricity. The stimuli were $3 \mathrm{~s}$ in duration and separated by 30 -s intervals. Each scan contained six repetitions and data were combined from six scans.

The HRF was derived as the response to a 1.5-s stimulus (the fMRI sampling rate). We used a $3 \mathrm{~s}$ rather than a 1.5-s stimulus because (a) linearity deteriorates at short stimulus durations (Birn et al., 2001; Boynton et al., 1996; Logothetis and Wandell, 2004) and (b) in our experiments the effective stimulus duration is $3 \mathrm{~s}$ or more. Therefore, deriving the HRF 
based on the 3-s stimulus will yield a more accurate prediction for our measurement conditions.

The responses to the 3-s stimuli were fit as the convolution of the responses to two 1.5-s responses, each of which is the HRF. We parameterized the HRF as difference of two gamma functions (Friston et al., 1998; Glover, 1999a; Worsley et al., 2002). This functional form of the HRF captures the late undershoot of the response better than a single gamma function (Boynton et al., 1996; Lange and Zeger, 1997). The percent variance explained (mean \pm S.D.) is $98 \pm 0.9 \%$ and $93 \pm 2.0 \%$ for the two-gamma and one-gamma HRF, respectively. The small difference has almost no effect on the estimated pRF; we computed these values using both HRF models and the results were in excellent agreement. The HRF (dashed line) and expected response to the 3-s stimulation (solid line) are plotted in Fig. 11.

\section{Appendix B. Limitations of traveling wave methods}

The traveling wave calculations assume that the peak fMRI response occurs when the expanding ring is centered on the pRF; this assumption can fail. Suppose we measure a simple pRF centered slightly to the right, but overlapping, the fovea (Fig. 12A). The magnitude of the fMRI response (arbitrary units) is denoted by the white curve shown on the image. The peak fMRI response occurs when the overlap between the ring and the $\mathrm{pRF}$ is highest, and this position differs from the ring location that covers the pRF center. The size of the deviation between the estimated and true eccentricity varies as a function of eccentricity and pRF size, as shown in Fig. 12B.

The simulations of Figs. 12A and B match the fMRI estimates plotted in Figs. 12C-D. These panels compare eccentricity estimates using the pRF model-based method and traveling wave methods. The data are divided into separate panels according to the receptive field size estimate from the pRF model-based method. We treat the pRF estimates as accurate and plot them on the horizontal axis. The traveling wave estimates are plotted on the vertical axis.

The data illustrate several points. First, the traveling wave eccentricity estimates are close to the smooth curves predicted by the theoretical calculations in Figs. 12A and B. Second, the eccentricity distortions do not appear in the estimates derived from the pRF model-based method. Third, the pRF model-based method estimates the positions of near foveal pRFs with large sizes (Fig. 12E), while the phase-encoded method fails.

\section{Appendix C. Analysis of HRF model errors}

We demonstrate that differences in the HRF model change the absolute - but not the relative - pRF size estimates. We varied the simulated HRF by perturbing the parameters of the optimal HRF fit by 30\% (Fig. 13A) for either the one- or the two-gamma HRF models (Boynton et al., 1996; Friston et al., 1998). This is a large parameter range, and in some instances the HRF parameter variations produces an uncharacteristic HRF profile. We included only HRF profiles with one positive peak and one negative peak with a total time course of maximal $30 \mathrm{~s}$. This perturbation yielded a total of $55 \mathrm{HRFs}$ that we used in the calculations (Fig. 13A).

Using each HRF, we estimated the pRF model parameters in V1 to V3. We calculated the average pRF size between $1^{\circ}$ and $13^{\circ}$ eccentricity for V1 to V3 from the best straight line fit (Fig. 13B). The average pRF size decreases as a function of the HRF width (full-width at half-maximum). This relationship is nearly perfect (average $\mathrm{V} 1-\mathrm{V} 3, \mathrm{r}=-0.98$ ) for the one gamma model (Boynton et al., 1996;Lange and Zeger, 1997), but not for the two gamma model (Friston et al., 1998; Glover, 1999a; Worsley et al., 2002) (average V1-V3, r=-0.26), 
we assume this is due to the variation of the late undershoot which is not characterized by the fwhm but will also affect the pRF size estimates. The relative pRF sizes, obtained by dividing the average pRF estimates of $\mathrm{V} 1$ to $\mathrm{V} 3$ by their mean for each HRF, are constant and independent of the HRF width (Fig. 13C). In all cases, average pRF size estimates in V2 and V3 are larger than V1 by about $40 \%$ and $100 \%$, respectively.

\section{Appendix D. Supplementary data}

Supplementary data associated with this article can be found, in the online version, at doi: 10.1016/j.neuroimage.2007.09.034. 
A

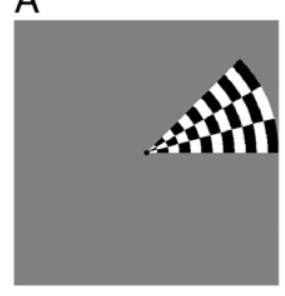

B

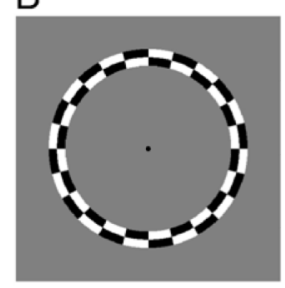

C

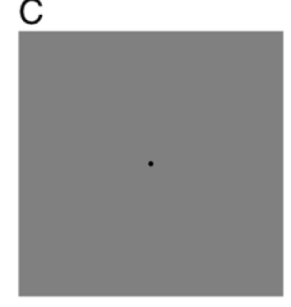

D

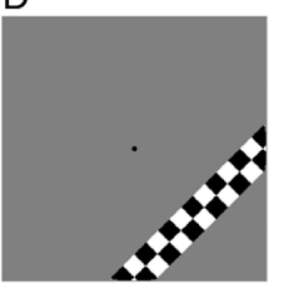

$\mathrm{E}$

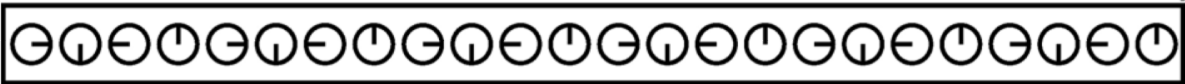

$\mathrm{F}$

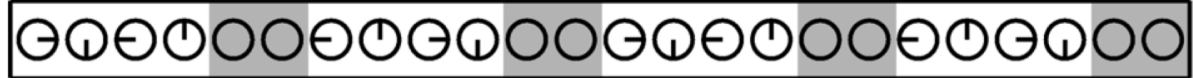

$\mathrm{G}$

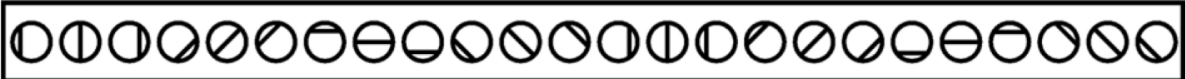

Fig. 1.

Illustration of the stimuli. (A-D) Static images of the wedge, ring, mean-luminance and bar stimulus, respectively. Panels E-G illustrate how the stimuli change within a single fMRI scan. (E) The wedge rotates through 6 full cycles per scan. (F) The rotating wedge with the mean-luminance blocks (gray regions); mean-luminance replaces the wedges at four intervals in time. (G) The bar moves through each of four orientations in two opposing directions during one scan. 


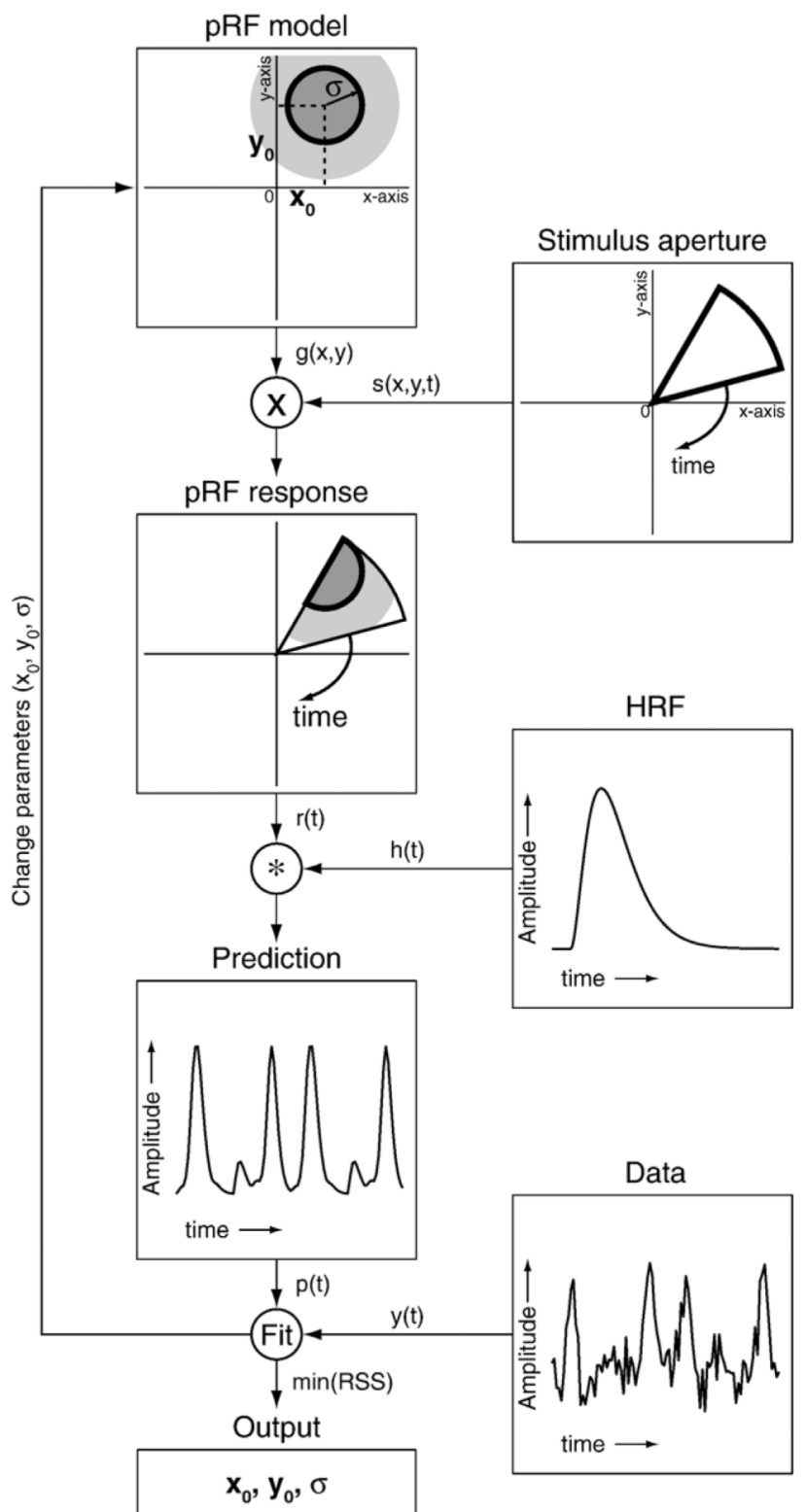

Fig. 2.

A flow chart describing the pRF linear model estimation procedure. The pRF linear model is calculated for every voxel independently. See text for details. 


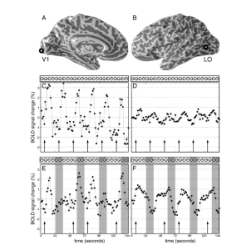

Fig. 3.

BOLD fMRI time-series from two voxels located in V1 (left panels) and lateral occipital cortex (LO, right panels). The circles in the top two panels (A and B) indicate the position of each voxel. The middle two panels $(\mathrm{C}$ and $\mathrm{D})$ illustrate the responses to a conventional rotating wedge mapping stimulus. The arrows indicate the wedge orientations that elicit the strongest fMRI response; the peak response is slightly delayed with respect to the presentation of the best orientation due to the hemodynamic lag. The two cortical locations responded best to similar wedge positions, but the percent BOLD modulation in V1 is about three to four times stronger than the modulation in LO. The bottom two panels ( $\mathrm{E}$ and $\mathrm{F}$ ) show the responses to the new stimulus with mean-luminance blocks inserted (gray regions). In V1, the insertion of mean-luminance blocks replaces some presentations of the preferred wedge position (first and third block) and hence no fMRI modulation is observed. In LO, the insertion of mean-luminance blocks causes a drop in the BOLD modulation, demonstrating that this LO voxel is responsive to all wedge orientations. 

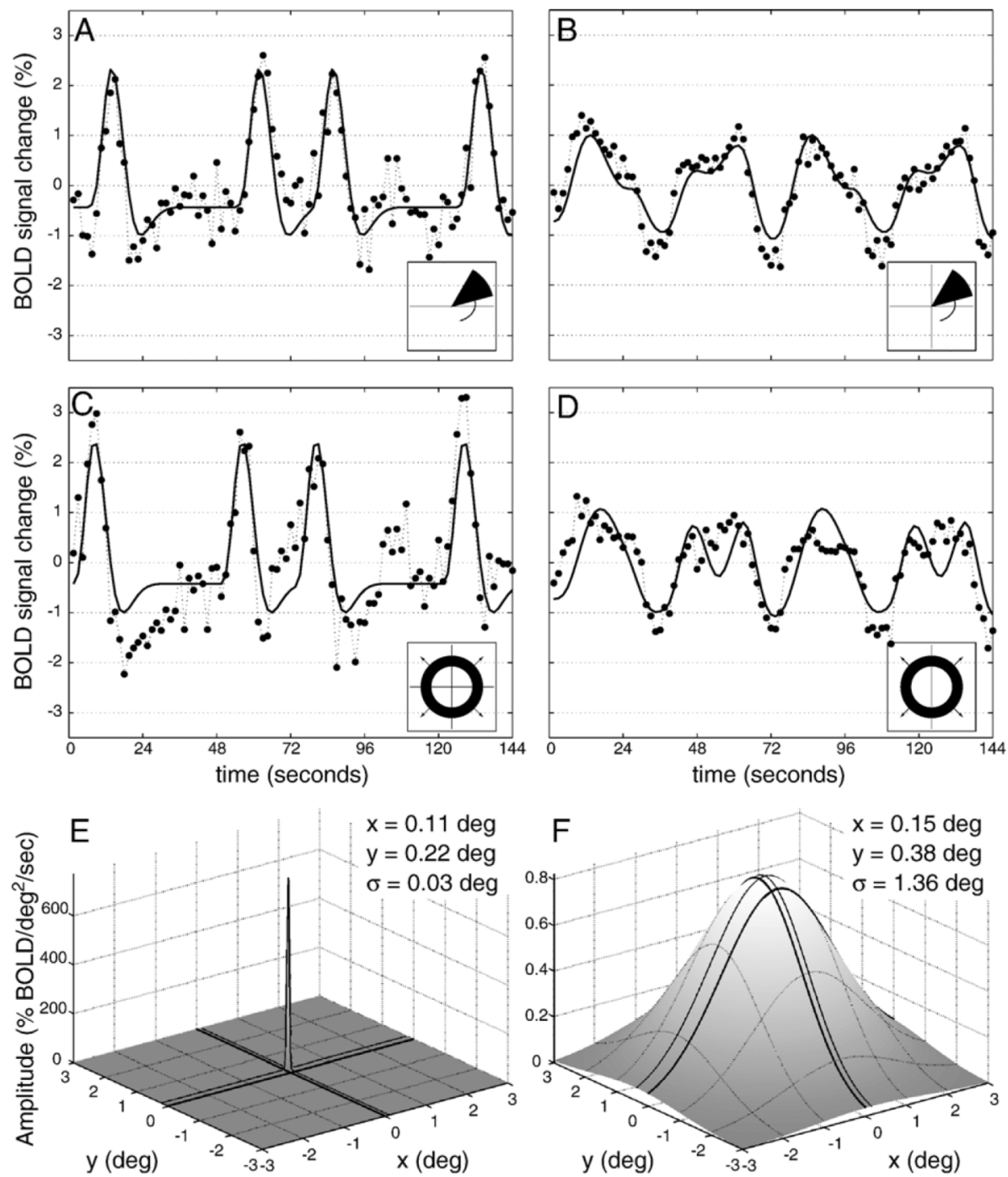

Fig. 4.

Example of a model fit to BOLD time-series at cortical samples in V1 (left panels) and LO (right panels, see Fig. 3). The model is simultaneously fit to the rotating wedge (A and B) and expanding ring $(\mathrm{C}$ and $\mathrm{D})$ data. The best fitting Gaussian isotropic model is shown in the bottom row $(\mathrm{E}$ and $\mathrm{F})$. The thick lines pass through the horizontal $(y=0)$ and vertical $(x=0)$ meridians, whereas the thin lines identify the $\mathrm{pRF}$ center. These models explain $72 \%$ and $68 \%$ of the variance in the time series, respectively. In panels A-D, the BOLD time-series are shown with dotted lines and black dots; the model fits are shown with solid lines (E and F). The response amplitude of the pRF models for a given stimulus is given by the integral within the stimulus aperture (see Fig. 2). 

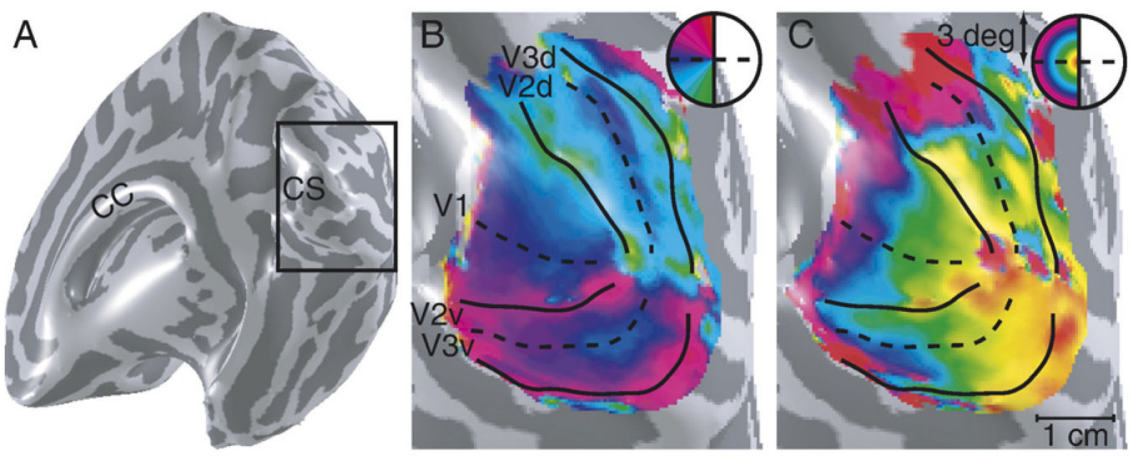

Fig. 5.

pRF position estimates on an inflated cortical surface. The corpus collosum (CC) and the calcarine sulcus (CS) are labeled to clarify the orientation of the inflated cortical surface. The position maps are displayed on the enlarged part of the occipital lobe, as indicated by the large black square in (A). The maps for polar-angle (B) and eccentricity (C) are shown. The insets indicate the color map that defines the visual field representation. The boundaries between V1 and V3 are identified by the solid black lines. 

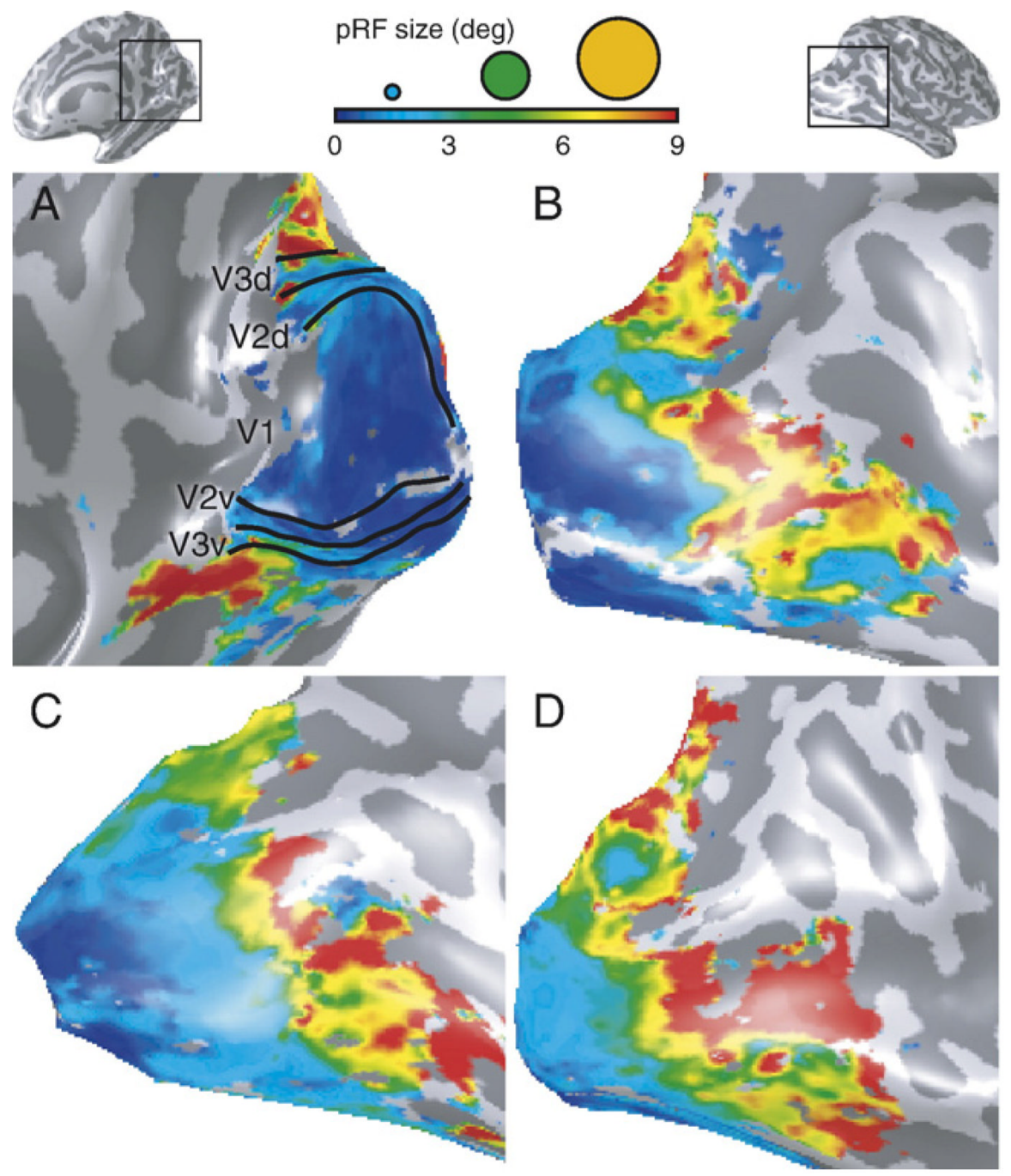

Fig. 6.

pRF size estimates on an inflated cortical surface. The pRF size maps are displayed on the enlarged part of the occipital lobe, as indicated by the black squares in the top two cortical surfaces. The colors indicate the different pRF sizes as shown in the color bar. The pRF size map for one subject is shown from the medial (A) and lateral view (B). The borders between visual field maps V1 and V3 are delineated by the solid lines. Lateral views from two additional subjects are shown in panels (C and D). The pRF sizes are much smaller in the maps near V1 than those on the lateral occipital surface. Supplementary Fig. 1 shows right, left, medial and lateral views for all three subjects. 


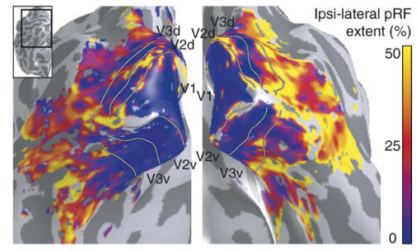

Fig. 7.

The percent of the $\mathrm{pRF}$ within the ipsilateral visual field is shown on an inflated cortical surface of one subject. The pRF in lateral (LO) and ventral (VO) occipital cortex overlaps substantially into the ipsilateral visual field. 


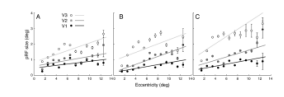

Fig. 8.

The relationship between eccentricity and pRF size in visual field maps V1-V3. Within each field, the pRF size increases with eccentricity. Furthermore, pRF size increases

systematically from V1 to V3. Separate panels are data from separate subjects. The solid lines are fit to the data (circles) within each visual field map. 


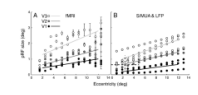

Fig. 9.

A comparison between fMRI-pRF (A) and electrophysiological receptive field sizes (B) in visual field maps V1-V3. The average fMRI-pRF estimates are indicated by the solid lines from Fig. 8; the average of single- and multi-unit receptive field size measurements from the literature are indicated by the solid lines (Burkhalter and Van Essen, 1986;Felleman and Van Essen, 1987;Gattass et al., 1981;Gattass et al., 1987;Newsome et al., 1986;Rosa et al., 1988,2000; Van Essen et al., 1984). We also plot LFP-pRF size and range estimates with a black square, white cross and dashed lines, respectively (Victor et al., 1994). 


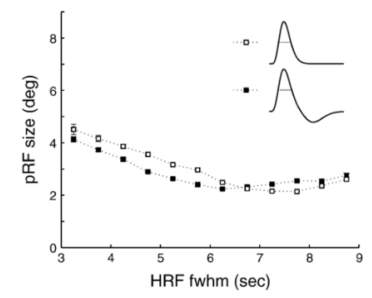

Fig. 10.

The average relationship of the HRF width and estimated pRF size. The horizontal axis represents the full-width at half-maximum of the HRF. The vertical axis represents the pRF size. The data are averaged from three subjects. There is a weak inverse relationship ( $r=$ -0.1 ), suggesting that HRF variations cannot explain the large estimated pRF variations. 


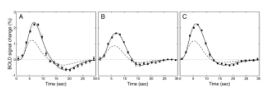

Fig. 11.

The average BOLD fMRI time series in V1 $\left(5^{\circ}-9^{\circ}\right)$ following a 3-s stimulus presentation. Each panel shows measurements from a different subject. The circles connected by the dotted lines show the measured data. The solid lines indicate the fit of the data with an HRF model using a difference of two gamma functions (Friston et al., 1998; Glover, 1999a; Worsley et al., 2002). The dashed line shows the HRF model prediction to a 1.5-s stimulus presentation. The HRF model explains $97.1 \%, 98.7 \%$ and $98.7 \%$ of the variance in the data (A-C, respectively). 


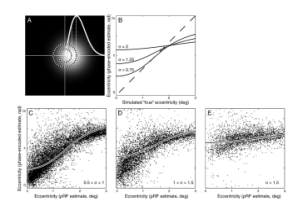

Fig. 12.

Illustration of the bias in pRF center estimates using phase-encoded methods. (A) The background image shows a theoretical pRF. The solid white line indicates the predicted BOLD response amplitude as a function of ring eccentricity. The maximal response is at a position slightly beyond the center of the pRF unit (large ring), not for the ring that overlaps with the center of the pRF (small ring). The phase-encoded method incorrectly takes the phase of the larger ring as an estimate of the pRF's center. (B) The graph illustrates the eccentricity estimates of the phase-encoded method ( $y$-axis) for different simulated pRF sizes $(\sigma)$. These phase-encoded estimates deviate increasingly from the true pRF eccentricity $(x$-axis) as a function of eccentricity and pRF size. (C-D) The three graphs show the eccentricity estimates of the pRF model-based method ( $x$-axis) versus phase-encoded method ( $y$-axis) of one subject. The three graphs are divided according to the pRF size estimates similar to the pRF sizes used in the simulation (panel B). These plots indicate that (i) the phase-encoded estimates are close to the smooth curves predicted by the theoretical calculations, (ii) the eccentricity distortions do not appear in the estimates derived from the pRF model-based method, and that (iii) the pRF model-based method estimates the positions of near foveal pRFs with large sizes, while the phase-encoded method fails. 

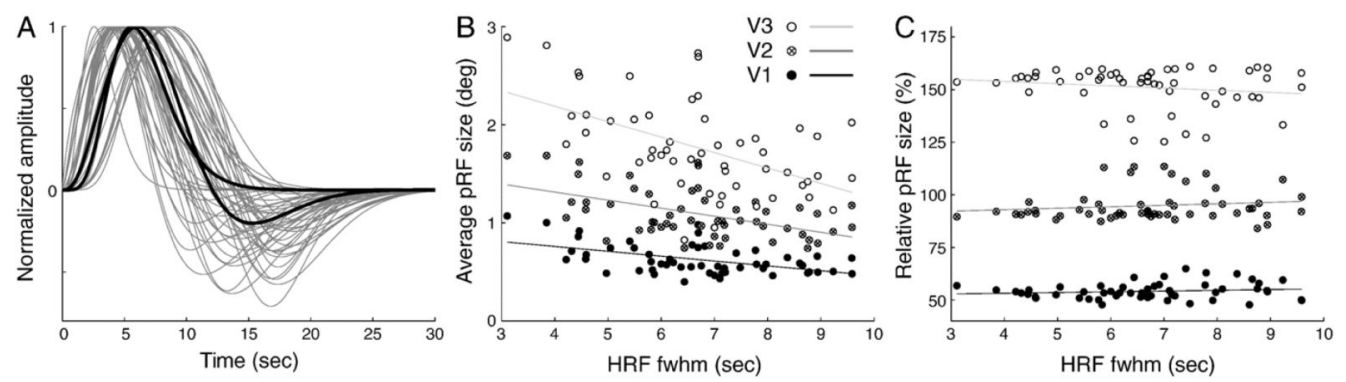

Fig. 13.

The relationship between the HRF model parameters and the pRF size estimates for the same dataset. (A) The different HRFs used to estimate the pRF parameters are shown. The HRFs are generated by a $30 \%$ perturbation of the optimal HRF (thick lines, see Fig. 11) of both the one- and two-gamma HRF model (Boynton et al., 1996;Friston et al., 1998). (B) We computed the average pRF size in V1 to V3 between $1^{\circ}$ and $13^{\circ}$ eccentricity for each HRF. The resulting pRF sizes are plotted as a function of the HRF width (full-width at halfmaximum, FWHM). Increasing HRF width yields decreasing pRF size estimates for the same data set. (C) The relative pRF size as a function of HRF width. The relative pRF size was computed by dividing the V1-V3 estimates by their mean for each HRF. The relative estimates are constant over a range of HRF width values. 


\section{Table 1}

Average correlation coefficient $(r)$ and standard deviation for three subjects

\begin{tabular}{lcc}
\hline Correlation coefficient $(\boldsymbol{r})$ & Polar-angle & Eccentricity \\
\hline Conventional stimuli & $0.97(0.01)$ & $0.87(0.08)$ \\
Stimuli with mean luminance & $0.97(0.01)$ & $0.84(0.10)$ \\
\hline
\end{tabular}

The correlation coefficient compares position estimates of conventional phase-encoded methods and the pRF model-based method. The correlation coefficient was computed for all voxels in the scanned cortex with coherence larger than 0.3 . 